\title{
Integration of VIRUS Spectrographs for the Hobby-Eberly Telescope* Dark Energy Experiment
}

\author{
J. Heisler ${ }^{\mathrm{a} \dagger}$, N. Mollison ${ }^{\mathrm{a}}$, I. Soukup ${ }^{\mathrm{a}}$, R. Hayes ${ }^{\mathrm{a}}$, G. J. Hill ${ }^{\mathrm{b}}$, J. Good ${ }^{\mathrm{b}}$, R. Savage ${ }^{\mathrm{b}}$, B. Vattiat ${ }^{\mathrm{b}}$, \\ ${ }^{a}$ The University of Texas Center for Electromechanics, 10100 Burnet Road - EME 133, Austin, TX \\ USA 78758- 4445; ${ }^{\mathrm{b}}$ The University of Texas McDonald Observatory, 1 University Station, C1402, \\ Austin, TX USA 78712-0259
}

\begin{abstract}
The Hobby-Eberly Telescope Dark Energy Experiment $\left(\operatorname{HETDEX}^{\ddagger}\right)$ at the University of Texas McDonald Observatory will deploy the Visible Integral-Field Replicable Unit Spectrograph (VIRUS) to survey large areas of sky. VIRUS consists of up to 192 spectrographs deployed as 96 units. VIRUS units are fiber-fed and are housed in four enclosures making up the VIRUS Support Structure (VSS). Initial design studies established an optimal array size and an upper and lower bound on their placement relative to the existing telescope structure. Tradeoffs considering IFU (optical fiber) length, support structure mass and ease of maintenance have resulted in placement of four 3 x 8 arrays of spectrograph pairs, about mid-point in elevation relative to the fixed HET structure. Because of the desire to minimize impact on the modal performance of the HET, the VSS is required to be an independent, selfsupporting structure and will only be coupled at the base of the telescope. Analysis shows that it is possible to utilize the existing azimuth drives of the telescope, through this coupling, which will greatly simplify the design and reduce cost. Each array is contained in an insulated enclosure that will control thermal load by means of heat exchangers and use of facility coolant supply. Access for installation and maintenance on the top, front, and rear of the enclosures must be provided. The design and analysis presented in this paper must provide an optimum balance in meeting the stringent requirements for science and facility constraints such as cost, weight, access, and safety.
\end{abstract}

Keywords: Center for Electromechanics, CEM, McDonald Observatory, MDO, Telescope tracking systems, HobbyEberly Telescope, HET, HETDEX, VIRUS, Visual Integral-field Replicable Unit Spectrograph

\section{INTRODUCTION}

The HET was originally envisioned as a spectroscopic survey telescope, able to efficiently survey objects over wide areas of the sky. While the telescope has been very successful observing large samples of objects such as quasi-stellar objects (QSOs) spread over the sky with surface densities of around one per 10 sq. degrees, the HET design, coupled with a small field of view corrector, hampers programs where objects have higher sky densities. In seeking a strong niche for the HET going forward, the HET field of view will be increased from ' 4 to 22 ' so that it can accommodate the Visible Integral-field Replicable Unit Spectrograph (VIRUS), an innovative, highly multiplexed spectrograph that will open up the emission-line universe to systematic surveys for the first time, uncovering populations of objects selected by their line emission rather than by their continuum emission properties.

The HET will undergo this major upgrade in 2011 to support HETDEX as well as current and future instrumentation. The HETDEX project consists of three primary elements:

- HET wide field upgrade (WFU) which includes designing, fabricating, and deploying a larger field of view wide field corrector (WFC) that will replace the existing spherical aberration corrector. It also requires design,

\footnotetext{
* The Hobby - Eberly Telescope is operated by McDonald Observatory on behalf of the University of Texas at Austin, the Pennsylvania State University, Stanford University, Ludwig-Maximillians-Universität München,and Georg-AugustUniversität, Göttingen

† JH Email: j.heisler@cem.utexas.edu

${ }^{\ddagger}$ http://hetdex.org/
} 
fabrication, and deployment of a new prime focus instrument package (PFIP) and tracker, as well as modification to the HET's azimuth bearings, to accommodate additional weight being added to the telescope.

- Design, fabrication and deployment of VIRUS on HET

- Execution of the Dark Energy Experiment ${ }^{1}$ (DEX) survey with the VIRUS on HET

The primary goal of HETDEX is to meet the DEX science requirements while preserving and/or improving upon HET's current capabilities. HETDEX science requirements were scrutinized at the Science Requirements Review (SRR) in June 2007. This was followed by a Preliminary Design Review (PDR) in April 2008 which scrutinized the technical requirements that "flowed down" from the science requirements. Both independent review panels found HETDEX to be scientifically compelling, technically feasible and programmatically sound.

A thorough review of the HET [Figure 1] was presented at the 2008 SPIE Astronomical Instrumentation Conference 2. This included a brief review of the HET so that the reader can better appreciate the differences between (1) the current and upgraded system designs, and (2) the differences between the HET and other more conventional alt-az telescopes. This review was followed by a high-level look at some of the more mature aspects of the system design as they were envisioned shortly after the PDR. Readers not familiar with the HET, WFU, HETDEX, and VIRUS are encouraged to review this 2008 paper because it contains pertinent background information that is not repeated in this paper. This paper focuses specifically on the VIRUS Support Structure (VSS), as well as the additional hardware required to support the 192 Spectrographs that make up VIRUS. More detailed information about VIRUS ${ }^{3 \text { thru } 12}$, and other aspects of the WFU ${ }^{13}$ thru 28 , is contained in several other papers that are part of the 2010 SPIE Astronomical Instrumentation Conference.

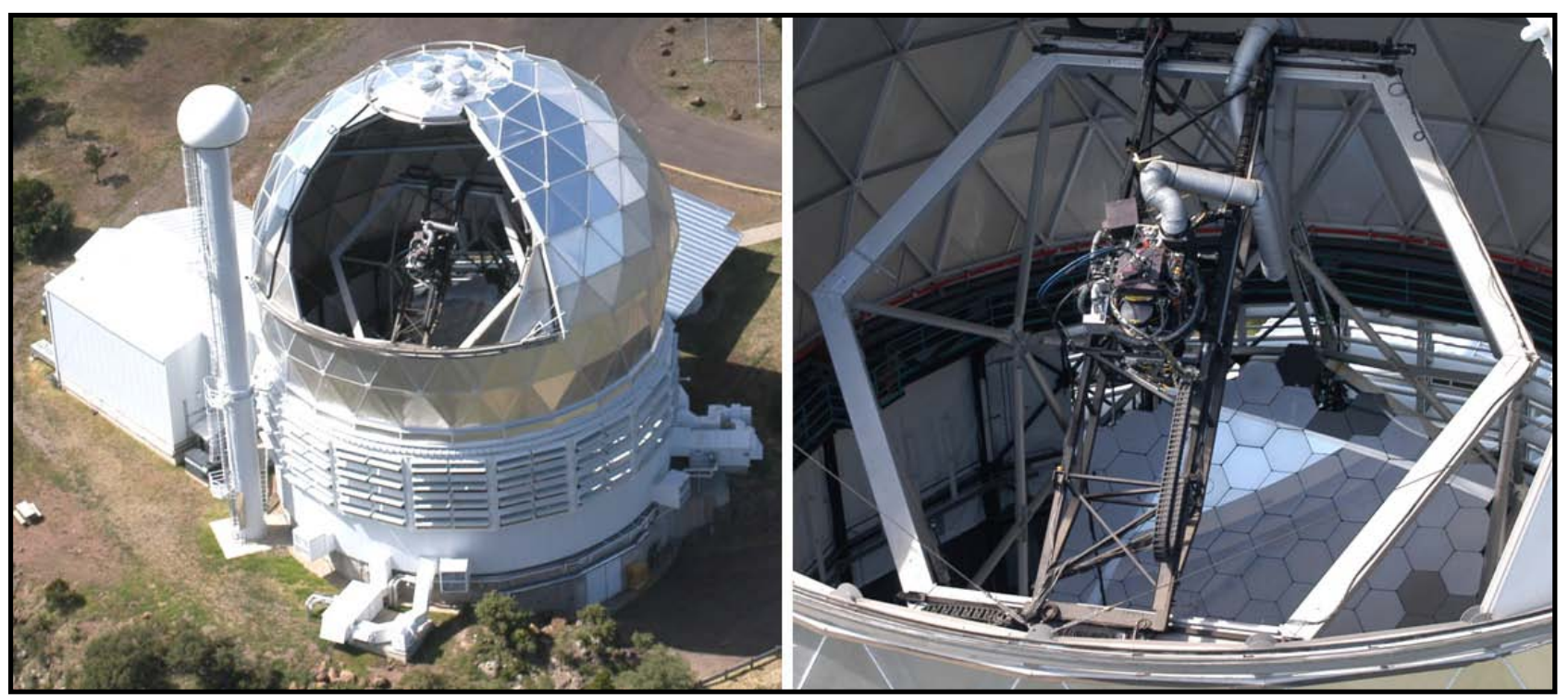

Figure 1. The figure on the left shows the HET pre WFU with the dome shutter open. The figure on the right is a close up view of the top of the HET revealing the primary mirror, and telescope structure which supports the tracker and

PFIP.

In addition to performing the engineering design and construction of the upgraded tracker for the WFU, the University of Texas Center for Electromechanics (CEM) has been tasked with integration of VIRUS to the existing HET structure. The subsequent sections provide a breakdown of the design goals for the VSS, and reviews the previous concepts that lead to the definition of those goals. These are followed by the current conceptual design of the VIRUS Support Structure (VSS) and related hardware, an overview of the preliminary analysis work, and a look at the path forward.

\section{CURRENT CONCEPTUAL DESIGN}

The location to support all of the spectrographs and ancillary hardware has been constantly evolving throughout the detailed design of the HET tracker upgrade. Major design objectives such as minimizing length of the VIRUS integral field units (IFUs), while at the same time minimizing support structure mass and cost, have appeared to be contradictory at times. The currently proposed location seeks to optimize the balance between these objectives. 


\subsection{Design Objectives}

As the design has progressed, four main goals have been identified as the main requirements for the VSS concept:

Minimize average IFU length, with a goal length of $20 \mathrm{~m}$ : This is driven by the need to diminish the transmission losses in the fibers before the light reaches the VIRUS spectrographs of wavelengths in the 350 to $680 \mathrm{~nm}$ range ${ }^{29}$. This is especially important for the lower wavelengths, as FRD testing on MDO's fiber optic test bench has demonstrated that lower wavelength transmissions are greater effected by FRD. Additionally a shorter IFU length corresponds to a smaller aerodynamic profile, which will help to minimize the wind loading on the fiber bundle.

Share the existing HET azimuth drive system, but isolate the VSS from the HET structure: An earlier trade study, ordered by MDO and discussed further in the following section, demonstrated that up to a certain total system mass, the current drive system is adequate to meet the desired acceleration rate of $0.25 \mathrm{deg} / \mathrm{sec}^{2}$. To keep the drive system as simple as possible, the VSS is tethered to the HET structure via kinematic links to join the VSS and HET structures during rotation on the azimuth. When the structures come to rest on their respective pads, the VSS should be sufficiently isolated from the HET structure so as to minimize any excitation due to wind. Wind loading poses a concern due to the large effective sail area (approximately $20 \mathrm{~m}^{2}$ ) of the largest side of each enclosure.

Minimize total system mass, with a maximum of $42000 \mathrm{~kg}$ : In addition to the requirement of maintaining a maximum weight for the acceleration rate on the drive system as per above, using FEA software to minimize structure mass while preserving stiffness will result in a higher overall natural frequency of the structure, reducing the likelihood of resonance within the structure due to wind excitation. The limit of $42000 \mathrm{~kg}$ stems from calculations based upon the $0.25 \mathrm{deg} / \mathrm{sec}^{2}$ minimum acceleration rate.

Allow adequate maintenance access to the mirror and tracker for cleaning and maintenance operations: Technicians at the HET facility use a telescopic man-lift to perform almost all of the maintenance operations required to keep the telescope in operation. This lift is required to access any portion of the tracker bridge or bearing rails, and it is used to reach through the structure and over the primary mirror in order to perform cleaning operations with compressed $\mathrm{CO}_{2}$. For these reasons it is of critical importance to avoid encroaching on the regions of the HET structure that the man-lift is threaded through during these operations not only to maintain efficiency during maintenance, but also to protect the structures from accidental damage from contact with the lift boom, and ensure the safety of maintenance technicians.

\subsection{Evolution Of The Concept}

In the time since the VIRUS support structure concept was introduced during the proceedings of SPIE Astronomical Instrumentation 2008, several iterations have been made on the fiber management system and the location of the VIRUS enclosures. Initial concepts for the VSS were developed in a trade study which clearly defined the upper and lower extreme boundaries for the enclosures [Figure 2]. While the low-mount concept offered low mass and simplicity of construction of the VSS, as well as the option to share the HET drive system, it also required the longest average IFU length. In addition, the VSS was rigidly linked to the base of the HET structure, presenting the possibility of transferring wind loading of the enclosures directly into the HET structure.
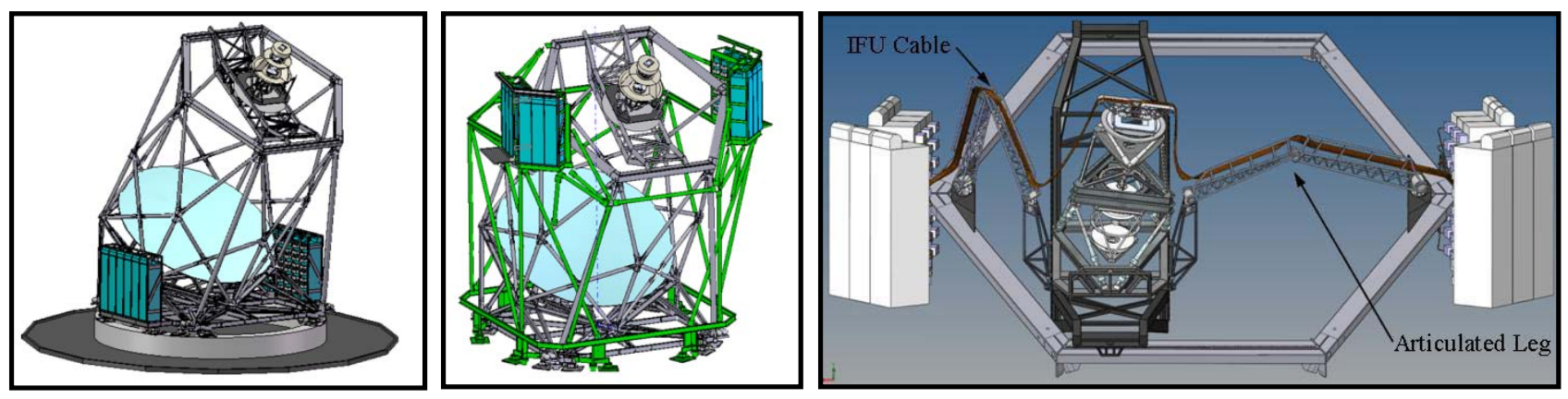

Figure 2. The images above left and center show, respectively, the low-mount and high-mount VSS designs and enclosure locations as of PDR. These concepts were presented in a trade study completed July of 2008. The image above right details the fiber management scheme for the high-mount design ${ }^{2}$.

The high-mount concept placed the enclosures in arguably the closest possible location to the tracker, in an effort to completely minimize IFU length. This came at a cost of a massive support structure which required a separate drive 
system, an industrial service elevator and additional platforms for maintenance access, and substantial additional modification of the upper hex to support the articulated leg IFU carrier. The IFU carrier was especially susceptible to wind loading, as it was located near the enclosure aperture. The design helped to determine the shortest possible IFU length, which was estimated at $15 \mathrm{~m}^{29}$. This study and the additional design work investigating the concepts allowed the overall design goals to be refined further.

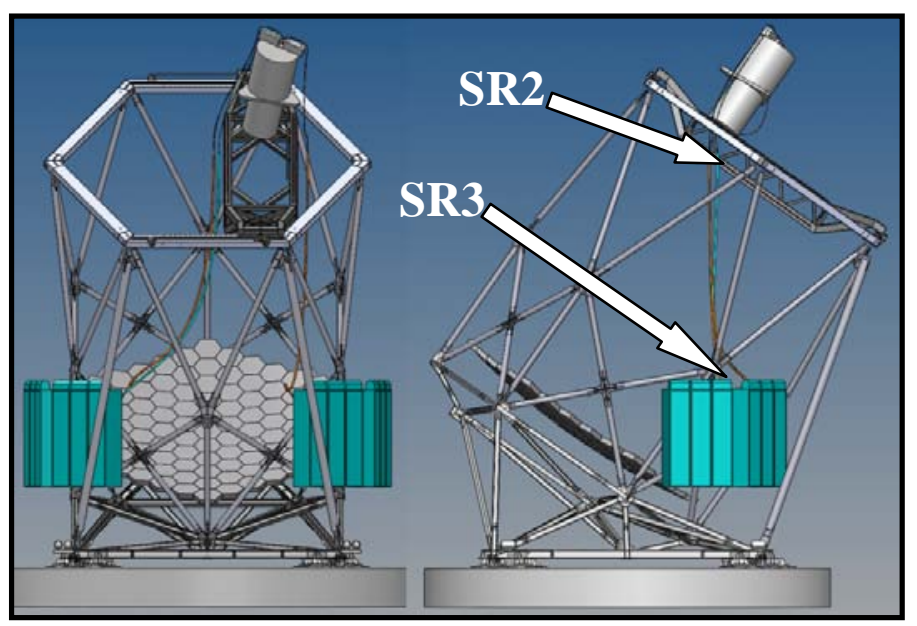

Figure 3. A mid-mounted enclosure location compromises between average IFU length and VSS complexity. This image shows the concept in late 2008, and the locations of the second and third IFU strain reliefs.
Shortly following PDR, investigation began into a mid-mounted enclosure location which would allow easy maintenance access to the enclosures while minimizing the average length of the IFUs [Figure 3]. In parallel, development proceeded on a test plan and hardware for accelerated life testing of the IFU bundles. With 448 fibers bundled together in each IFU, and the IFUs bundled into a group of 48 for each side of the telescope, replacement of individual fibers is not technically feasible. This testing will validate that there are no ill effects to the individual fibers when loaded in tension from their own weight due to gravity, as there is a concern of cracking, breakage, or FRD with the long, unsupported IFU runs inherent to this enclosure location ${ }^{29}$. Engineering design of the IFU testing mechanism has been completed and the system is currently under construction at $\mathrm{CEM}^{5}$.

\subsection{Arrival At Current Location}

As the design progressed, the HET Operations Staff discovered that the mid-front location significantly obstructed areas of the structure used for man-lift access. A side-mounted enclosure location was proposed, and MDO performed a study at the HET facility to determine whether new final strain relief points (SR3), located at either side of the structure and almost on the centerline, would negatively impact the IFU runs from the strain relief mounted to the tracker bridge (SR2). A physical representation of the IFU conduit bundle was hung between SR2 and SR3 and the tracker was then moved to each of the limits of travel, to ensure the fiber length was adequate, as well as visualize the route [Figure 4].

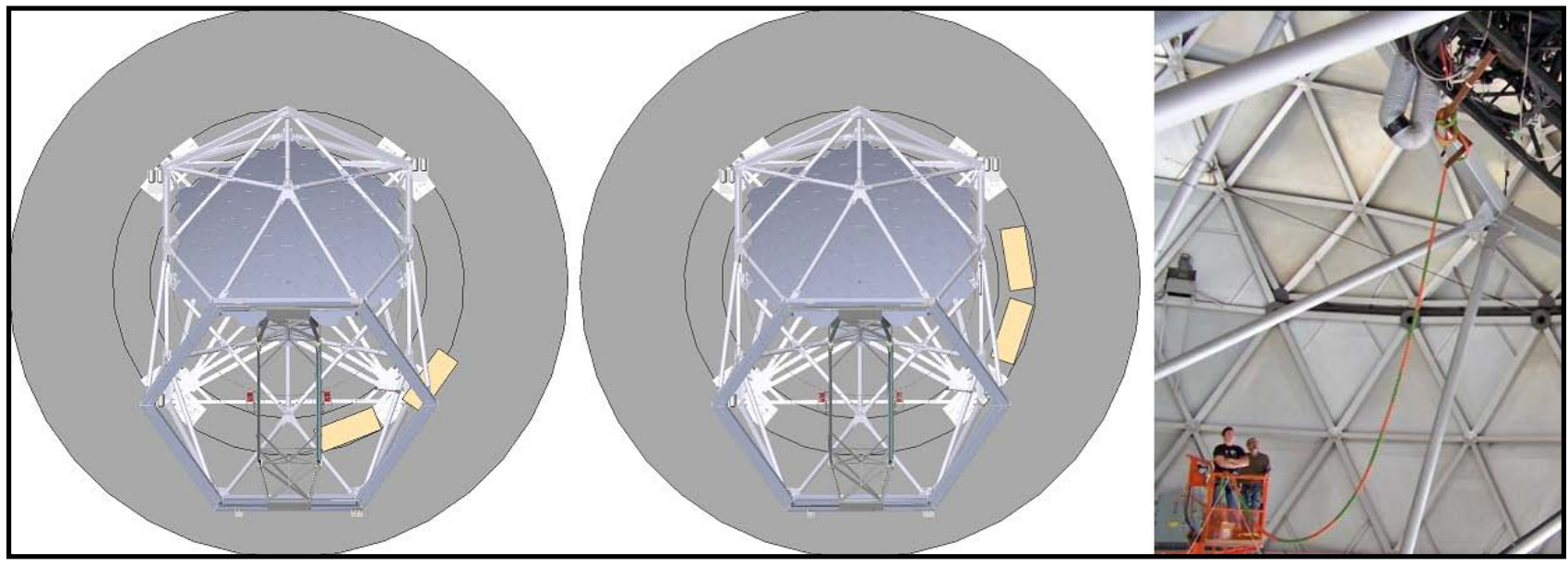

Figure 4. The front and side VIRUS enclosure locations were examined during a study that provided the first physical representation of the route of the IFU conduit bundle outside of CAD. Both of these potential enclosure locations locate the enclosures mid-way up the telescope, with the bases approximately $7 \mathrm{~m}$ above the pier.

The study showed that while a side mounted location of the enclosures forced an increase in length of the IFUs by half of a meter, it provided more favorable routing of the IFU bundle. The slack portion of the bundle, present when the 
tracker moves closer to the enclosures, droops less at the limits of tracker movement in this configuration [Figure 3]. In the front-mounted location, when the tracker moves to one of the lower extents, it is located almost directly over SR3. This imposes a very tight bend radius on the drooping portion of the IFU bundle, and also forces it to enter SR3 at a very sharp angle. The gradual slope of the route into the strain relief is preferred in order to minimize the potential for damage to the fibers. For this reason, as well as the greatly increased maintenance access to both the VIRUS arrays and other portions of the telescope, the side location is employed in the current concept and IFU test plan.

\subsection{Current Support Structure Concept}

The VSS is composed of two symmetric, free-standing structures located on either side of the primary mirror, supported and linked to one another by two trusses [Figure 5]. Average IFU length with the enclosures located on top of these structures is approximately $23 \mathrm{~m}$ total, which exceeds $20 \mathrm{~m}$ goal length. However, the current IFU length from the tracker to SR3 is estimated at $17 \mathrm{~m}$, so this equates to an average of only $6 \mathrm{~m}$ from SR3 to the collimators.
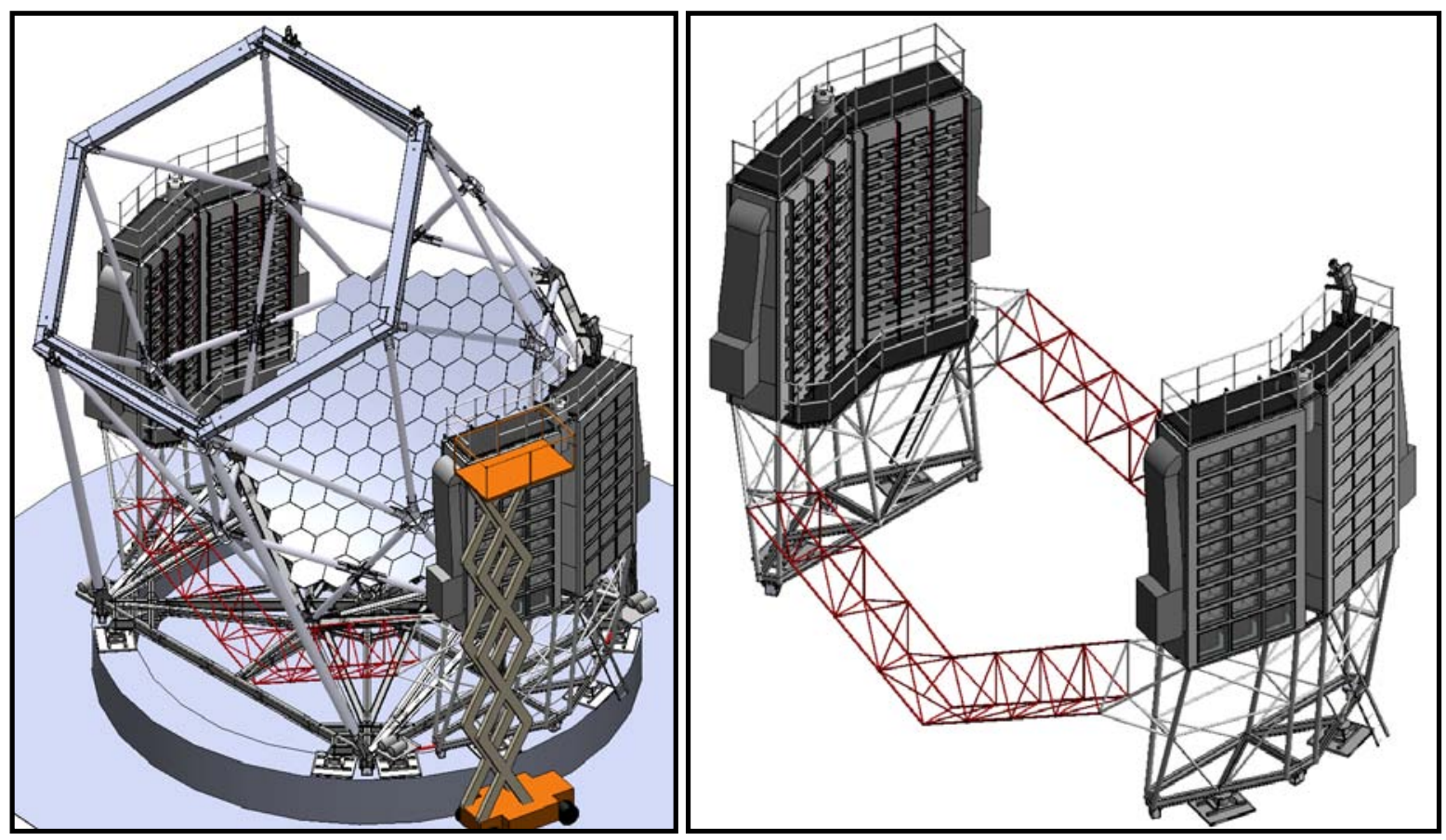

Figure 5. The current VSS concept with the tracking package removed for clarity.

A36 structural steel will be used extensively in the construction of the support structure and the enclosure frames, however other enclosure components and the work platforms may be composed of aluminum in order to minimize mass above the lower work platform. Reducing mass in this area could help avoid any wind-induced resonance at the tops of the enclosures, which preliminary analyses have shown to be the greatest affected areas. The vertical load bearing beams beneath each enclosure are 5" schedule 40 pipe, and these are tied together with 8" i-beams at the base and 4" channel under the enclosures. The structures are further reinforced with lengths of 2" pipe connecting major nodes. Each structure is supported at the base by three corner post assemblies incorporating non-marring rubber pads to prevent damage to the pier, identical to those currently supporting the HET.

Trusses, highlighted in red in the figure, provide stability for the structures when rotating on the azimuth or when subjected to transient wind loading. Composed of 2" and 1.25” schedule 40 pipe, the trusses weave through the existing HET structure, linking the two sides of the VSS without rigidly attaching to the HET.

The triangular cross section of the front truss is oriented in a manner that maximizes clearance for the telescopic boom of the man-lift in the front of the mirror [Figure 6]. During mirror cleaning operations, the boom of the man-lift runs almost parallel to the primary mirror as the operations staff cleans the surface with a jet of compressed $\mathrm{CO}_{2}$. To avoid the possibility of contact with the man-lift, the front truss wraps around the large I-beams at the base of the HET to provide maximum clearance. The rear truss is reversed to minimize intrusion into the area behind the mirror, which currently is occupied by facility hardware. 

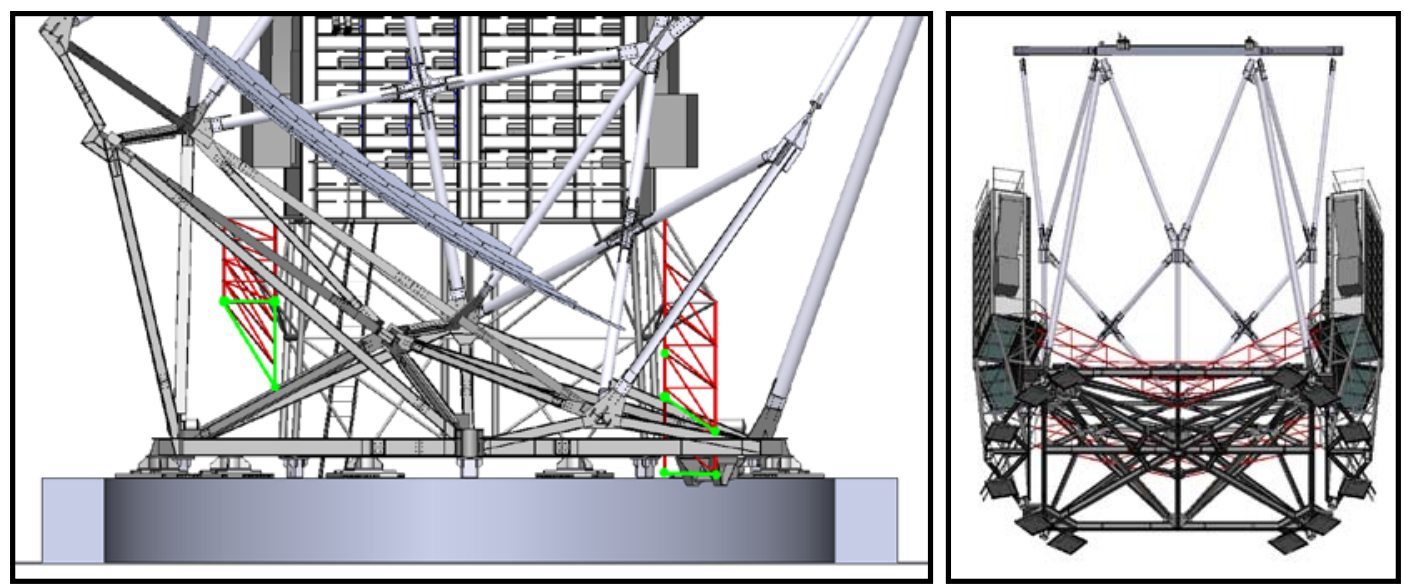

Figure 6. The left image shows a cross-section of the base of telescope with the trusses highlighted where they intersect the section plane. The image on the right is a view from below the front of the telescope showing the clearance allowed by the front truss.

\subsection{Rotation on the Azimuth}

Air bearings, tied to the existing air bearing system on the HET, will support the vertical load of the structure during rotation on the azimuth drive. There is adequate space beneath each side of the VSS for two or four bearings between the corner posts, so the additional stability of the extra bearings may outweigh the additional cost. Interlocks built into the control scheme use sensor data at each bearing location to prevent rotation should some of them fail to inflate. The structures are located and driven by horizontal kinematic linkages that attach only the bottom corners of the VSS to the rigid corners of the HET base. Tentative locations are represented in Figure 5, near the air tanks at the corners of the structure. The hardware must sufficiently isolate the HET from any transmitted vibrations, and as such may require some mechanism to automatically decouple the structures following a rotation.

\subsection{Enclosure Design}

Four large (6m height, 3m width, $1.4 \mathrm{~m}$ depth) custom-designed enclosures house VIRUS in $3 \times 8$ arrays. Other major system components within the enclosures include a liquid nitrogen distribution pipe network for the cryogenic system used to cool the cameras, and three $12 \mathrm{U}$ server racks located within a thermally isolated lower compartment of the enclosure. The server racks will accommodate all spectrograph detector electronics, as well as enclosure data acquisition systems, which are used to monitor the enclosure environment and cryostat vacuum transducers.

To reduce the amount of particulate matter introduced to the sealed enclosures when performing maintenance operations, the enclosures have a constant instrument air purge, and access to either side of the collimators of the VIRUS spectrographs is provided through individual doors mounted to the front or rear main enclosure panels. All access doors will provide a light-tight seal and be insulated to the same degree as the rest of the enclosure. Once maintenance is complete and the access door is replaced, filters located in the air handling units will remove any airborne contaminants.

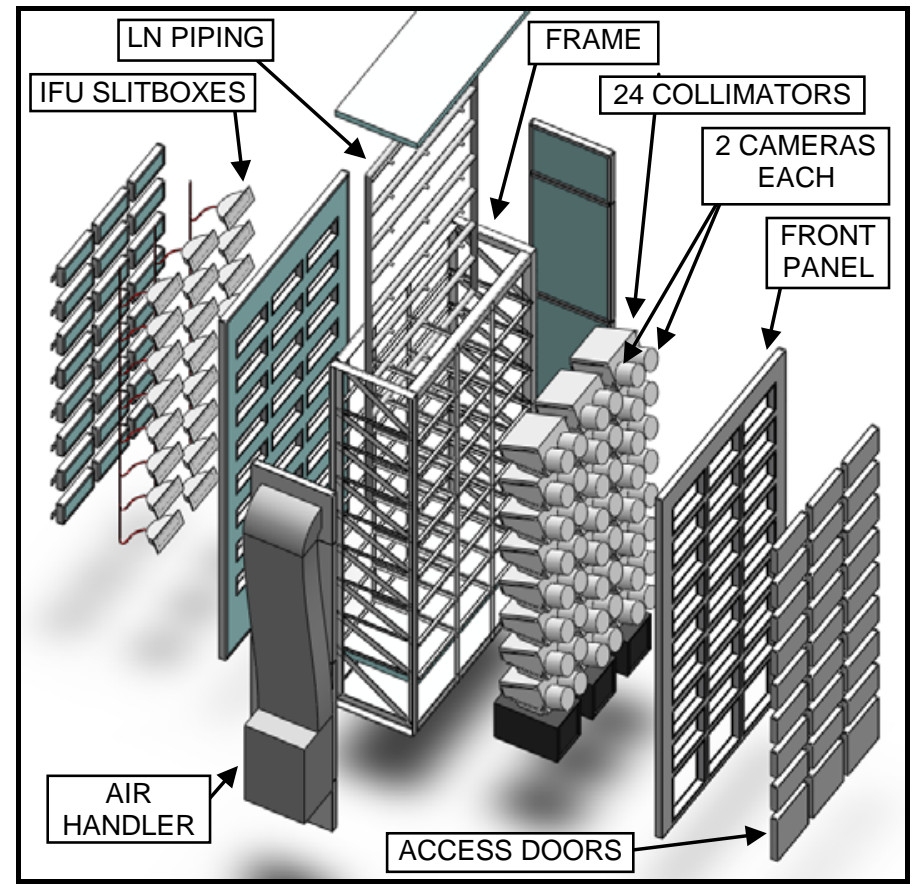

Figure 7. Enclosure Exploded View 
The exploded model of the VIRUS enclosures illustrates the assembly order of the individual components [Figure 7]. Each enclosure begins as a basic frame of welded structural steel, with the internal bracing removed in order to receive the liquid nitrogen distribution piping. The insulation at the bottom of the enclosure, as well as the insulation isolating the spectrograph compartment from the server rack compartment, is installed at this time. The liquid nitrogen pipe array is fed into the enclosure from the top and internal support braces are bolted into place. The support braces serve to both stiffen the enclosures and provide mounting points for the hardware required to constrain the spectrographs. Next the collimators and server racks are installed through the front of the enclosures, and top, rear, and side panels are installed, along with air handling units. At this point the server racks are populated, cameras are installed on the collimators, and all necessary cable and hose routing is completed within the enclosure. With the internal assembly completed, the front panel is installed with individual access doors in place.

If an external crane is available on site at this point in the assembly process, the enclosures will then be lifted in to position on the VSS and bolted in place. If the schedule does not allow this, the enclosure frames must be fitted to the VSS before installing the collimators, due to the limited capacity of the internal dome crane, and the rest of the installation will proceed at height. The final installation step involves attaching each of the individual IFU slitboxes to their respective collimators, and the methodology for this is discussed in a following section.

\subsection{Enclosure Thermal Management}

Due to the substantial surface area of the enclosures, there is a risk of dissipating excessive heat into the dome through the enclosure skin due to the elevated temperatures within. Internal temperature will be controlled via closed loop cooling utilizing chilled facility coolant, and the floating set point will correspond to the current ambient conditions. The thermal management systems must have the capacity to efficiently remove $3 \mathrm{~kW}$ from each enclosure, $30 \%$ of which is generated in the spectrograph compartment. To minimize the thermal signature of the enclosures due to varying internal temperatures, a minimum of 4 " of insulation on all sides has been incorporated in to the concept models. Initial estimates of insulation thickness assume usage of expanded polystyrene foam. Alternate materials may provide similar performance with reduced thickness. While the goal of the enclosure thermal management system is to maintain interior temperature at ambient, some of the hardware in the server racks is not rated for operation below $0^{\circ} \mathrm{C}$. Marginal operation for the telescope includes a temperature range of -10 to $25^{\circ} \mathrm{C}$, so temperature inside the server compartment will be held at just above freezing when ambient temperature drops below $0^{\circ} \mathrm{C}$.

Air handling units mounted to the sides of the enclosures employ high-flow centrifugal blowers to draw enclosure air through a filter and cooling coil at the base of the VIRUS compartment before returning it to the top of the enclosure in a closed loop [Figure 8]. Several manufacturers offer off-theshelf fan coil designs for HVAC use which incorporate all of these components in a compact package, which should help to minimize the amount of design time required for the system. Ductwork at the top of the enclosure will likely be required to evenly distribute the chilled supply air to all portions of the compartment and eliminate localized areas of increased temperature. By drawing return air from the bottom and returning chilled air to the top, the mass of the air handling unit is kept closer to the rigid VSS, and maintenance access for the fan, coils, and filters can be performed with the man lift at a lower and possibly more stable position on the lift.

Due to space constraints and to simplify the design, the thermally isolated server compartments below the VIRUS compartments will share a single air handling unit with the neighboring enclosure. The air handling unit will be located beneath the enclosure platform on the VSS and ductwork will link the two enclosures [Figure 8].

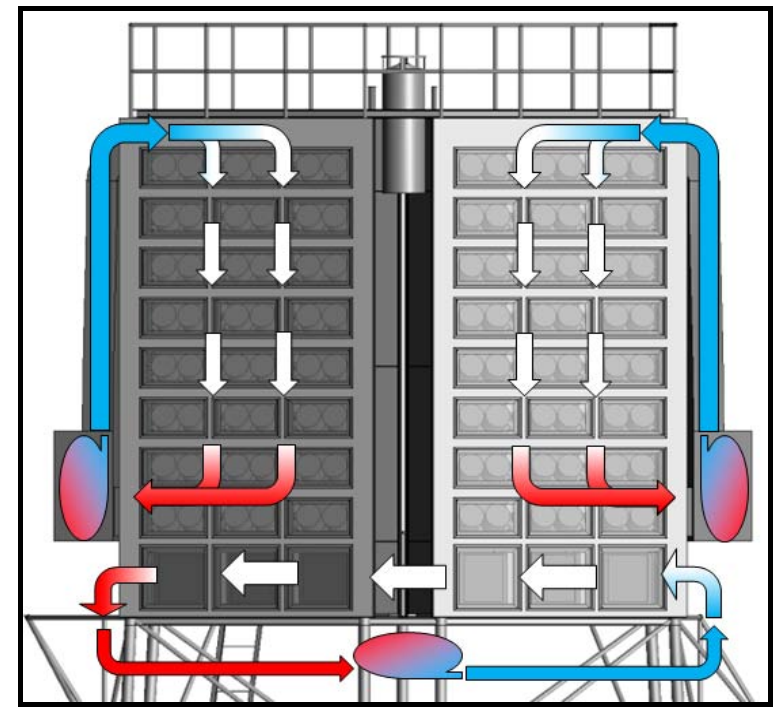

Figure 8. Arrows indicate air flow through the enclosure thermal loops. Hardware for the server rack compartments has not yet been modeled, but the desired flow path is indicated.

The DAQ system in each enclosure will ensure that the thermal management system is functioning properly by measuring air temperature at different locations within and on the skin of the enclosure, measuring coolant inlet and 
outlet temperature, and detecting any coolant leaks around the cooling coils before damage can be done. To avoid any type of a thermal gradient within the enclosure, the blower fans will run constantly, and coolant flow to the cooling coils will be regulated via servo valves fed by control signals from the DAQ hardware.

\subsection{Work Platforms and Ladders}

Maintenance procedures include IFU slitbox installation and removal on the inner (mirror) side of the enclosures, and routine vacuum pumping of the VIRUS camera cryostats on the outer side of the enclosures. Technician access to the outer side of the enclosures is provided via a scissor lift permanently located inside the dome [Figure 5]. Foundation reinforcements are required to support the lift, therefore it will remain stationary while the HET is driven on the azimuth to reach each individual enclosure. The lift provides an excellent means of safely transporting the vacuum pumping station, which weighs approximately 60 pounds, to mounting points on the enclosure. Access panels to the VIRUS cameras, as well as the server racks in the lower compartment, are all within an arm's reach while the technician remains safely restrained inside of the man-lift. Side-mounted air handling units are offset toward the outsides of the enclosure in order to facilitate easier routine filter replacement as well as motor or coil maintenance.

Work platforms linking the tops of the two enclosures on one side of the telescope are also accessed by way of the scissor lift. This platform allows service to the liquid nitrogen dewar and distribution hardware, and provides a location for a technician on the platform to assist a technician on the ladder in mating the IFU slitboxes to the collimators. This procedure is discussed in further detail in the following section. A ladder starting at the pier provides access to the inner work platform [Figure 9]. In order to minimize the cantilever effect on the VSS, the enclosures are placed in fairly close proximity to the HET structure, and as a result space in this area is limited. For this reason, platform entry is gained via a trapdoor, which also serves to prevent a possible falling hazard once the technician is standing on top of the platform. A grid of ladder rungs covers the inner sides of the enclosures, which provides technician access to the rear of the collimators for IFU installation.

\subsection{IFU Installation and Protection}

A detailed procedure for IFU installation, from the top of the tracker to the collimators, is still in development. Care must be taken so as not to damage the delicate fibers as the IFU conduits are uncoiled from the final strain relief and laid into place. The current concept is to route the IFU conduits from the final strain relief to cable trays running down the length of the inner enclosure ladders [Figure 9], which will provide support along the route as well as protection from accidental damage by staff on the ladders. After entering the tray, the IFU conduit will be routed down to the level of its respective collimator where it will split off into a strain relief in the rear access panel, and then continue to the IFU.
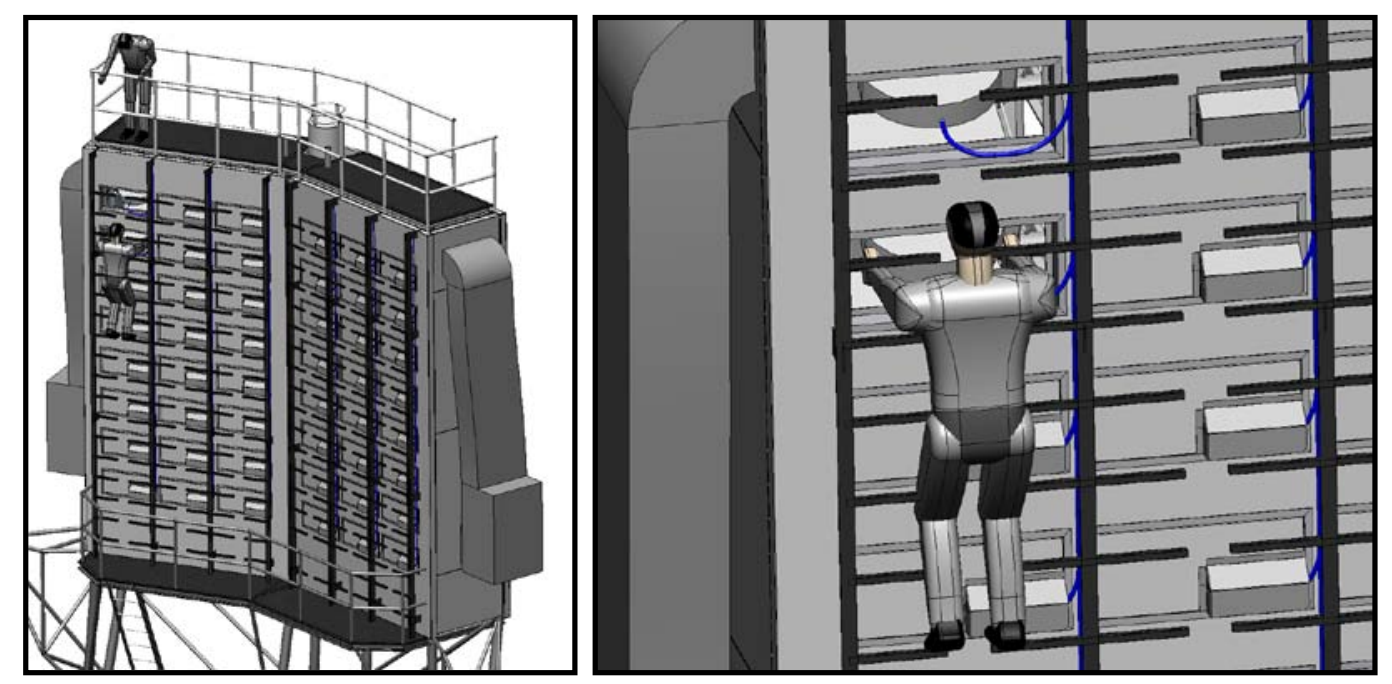

Figure 9. The IFU slitbox installation procedure requires one technician on a ladder to mount the slitbox, and one technician on the top platform to guide it into place with a hoist. Safety restraints are not shown in the image but will attach to the technician's harness at approximately waist high and allow him to work with both hands.

Installation of the IFU slitbox requires a coordinated effort between two technicians, one on the ladder accessing the back of the collimator to attach the slitbox, and one operating a hoist to safely direct the slitbox to the corresponding 
access panel. After scaling the ladder with his harness attached to a lifeline, the technician will attach restraints on his harness to the ladders to safely allow use of his hands. He will then remove the access panel to the collimator and move it out of the way through the ladder rungs. The access panels will incorporate a feature to allow hanging them from the ladder at height for storage while the technician works inside the enclosure. At this point the slitbox is inserted in to the enclosure and fastened to the collimator. The IFU conduit is then routed through the center gap between the rungs and captured within the protective cable tray. Finally the access panel, which incorporates a strain relief and environmental seal around the conduit, is reinstalled.

\section{CURRENT ANALYSIS WORK}

The VSS is currently modeled in Solidworks using structural member elements, which are essentially extrusions of a selected weldment profile, such as square tubing or channel iron, along a straight sketch path. Since the detailed connections between structural members are not necessary for analysis at this point in the design process, modeling in this fashion allows rapid iteration on Solidworks Simulation analyses to optimize the structure shape. During analysis each structural element is treated as a beam and sectioned into a finite number of elements in the mesh. Once loads are applied, the solver calculates axial and bending stresses at each segment in the beam mesh, and these stresses are checked against material yield limits.

\subsection{Structure Static Analysis}

Two basic static analyses were run with gravity applied as the load; one with "immovable" constraints at the three corner posts of each side of the VSS, and another with the constraints at the two air bearing locations on either side of the structure. This constraint allows rotation but prevents translation, so the assumption is that friction will prevent the pads from sliding, but the structural members could pivot at those points under load. The two cases represent the loading of the structure when supported solely by the corner posts, and solely by the air bearings. Due to issues observed on previous simulations using mixed meshing, only the components modeled as structural members are included in the analysis. To accurately represent the masses of the enclosures, including spectrographs and other hardware, a mock density was applied to the enclosure frame material to represent the full weight of the enclosure. Each enclosure is estimated at a mass of $4500 \mathrm{~kg}$.
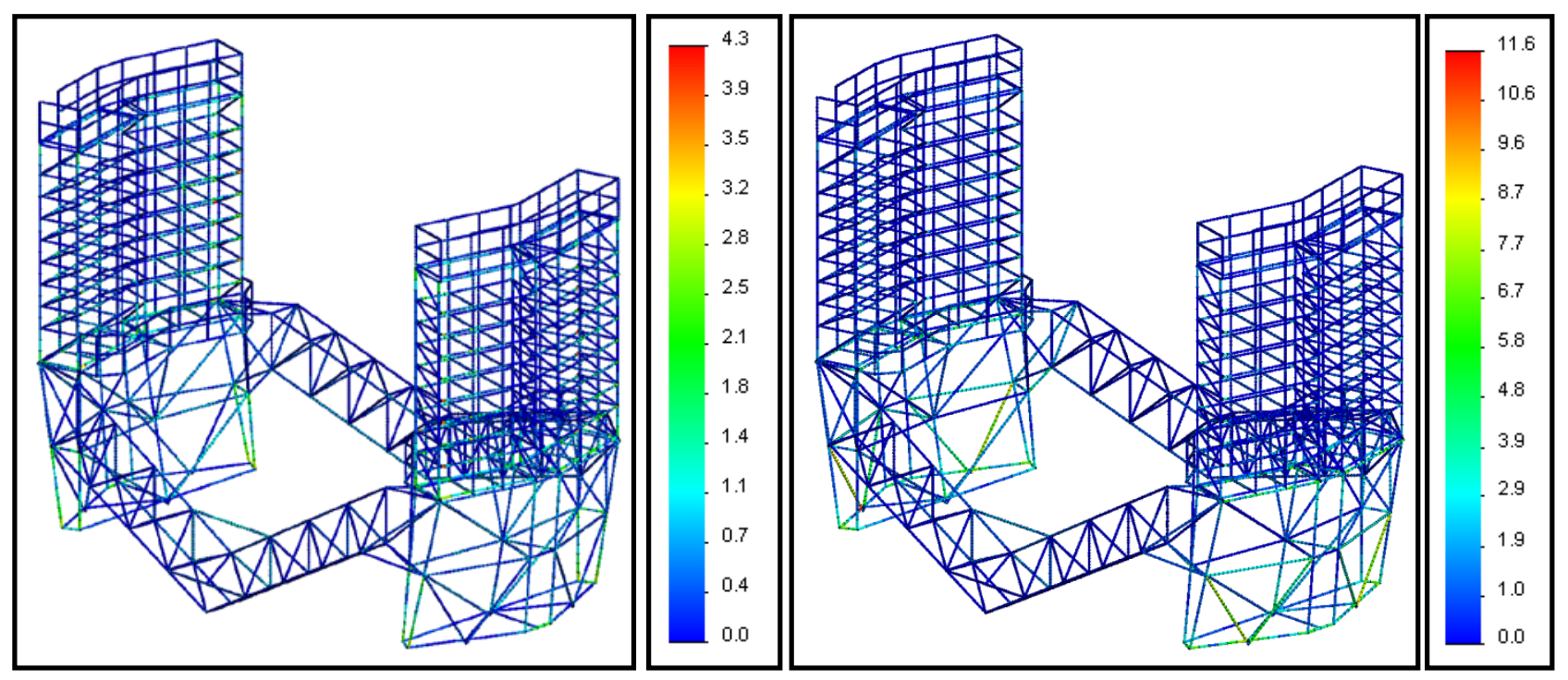

Figure 10. The left image shows the static analysis with constraints at the corner post assemblies, while the right image shows the same analysis with constraints at the air bearings. The stress scales are listed in units of ksi.

When resting on the corner posts, the maximum stress in the VSS itself is $3.2 \mathrm{ksi}$, corresponding to a safety factor of over 10 for A36 steel, and it is localized to the steel directly supporting the weight over the corner posts [Figure 10]. When supported by the air bearings, maximum stress is $11.6 \mathrm{ksi}$, corresponding to a safety factor of 3 , and is located at the air bearing nodes. While this stress is significantly below the fatigue limit of the steel, these nodes will be reinforced to ensure that the safety factor throughout the structure is a minimum of 5 . The structure will be optimized further, but 
currently sits at an approximate mass of $7000 \mathrm{~kg}$, including each side structure, front and rear trusses, and work platforms.

\subsection{Structure Modal Analysis}

The same basic model setup as described in the static analysis section above was used for the modal analysis of the structure. The natural frequency of the entire assembly is $1.8 \mathrm{~Hz}$, which is near the point where wind excitation can have significant effect. $2.0 \mathrm{~Hz}$ has been targeted as a goal minimum natural frequency.

The mode shape plot indicates swaying at the tops of the enclosures [Figure 10]. Efforts will be made to stiffen the enclosures, but given the minimal amount of deflection in the lower parts of the structure where the kinematic mounts to the HET are located, this may be adequate to avoid transferring load. Displacement at the top of the structure should be minimized within reason for worker safety on the top platform and inner ladders.

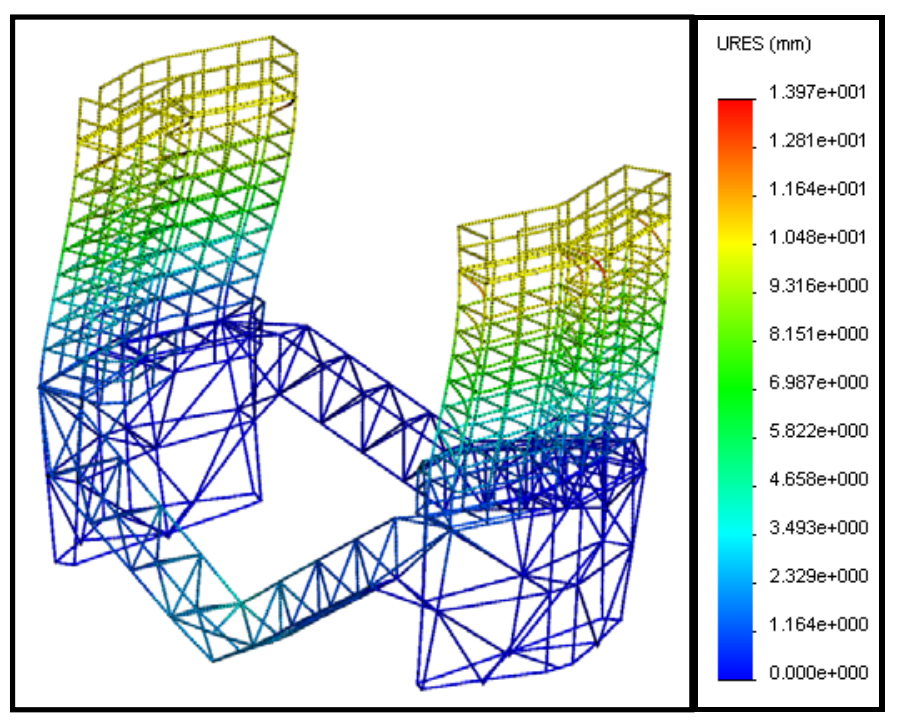

Figure 11. Mode shape of $1.8 \mathrm{~Hz}$ natural frequency

\subsection{Truss Static Analysis}

Initial investigation into sizing the trusses has shown that the worst case for stress within the truss is when the structure is only supported by the air bearings. Rotation of the structure on the azimuth was investigated, but the angular velocity is maintained at $3 \%$, and the loads on the trusses due strictly to this rotation are minimal. While the VSS is resting on the corner posts, as long as the CG of each side of structure and its enclosure pair is located between the three contact points on each side, almost the entire load is directed into the pier. The structures bear no moment about the contact point with the pier, and minimal load from the structure is supported through the truss. When the air bearings are inflated, however, each side of the structure is supported on only two contact points, and will bear a significant moment depending upon how far the CG is from the air bearing nodes.

While the current model shows that the CG of each side of the structure is supported almost directly above the air bearing nodes, an initial analysis was made assuming a worst case of $+/-0.75 \mathrm{~m}$ out of alignment. This corresponds to the maximum displacement of the CG within the three contact points of the corner posts, or the point at which each side can no longer freely stand without the trusses. This maximum misalignment would cause the trusses to be loaded with 28 $\mathrm{kN}$ of compressive or tensile force.
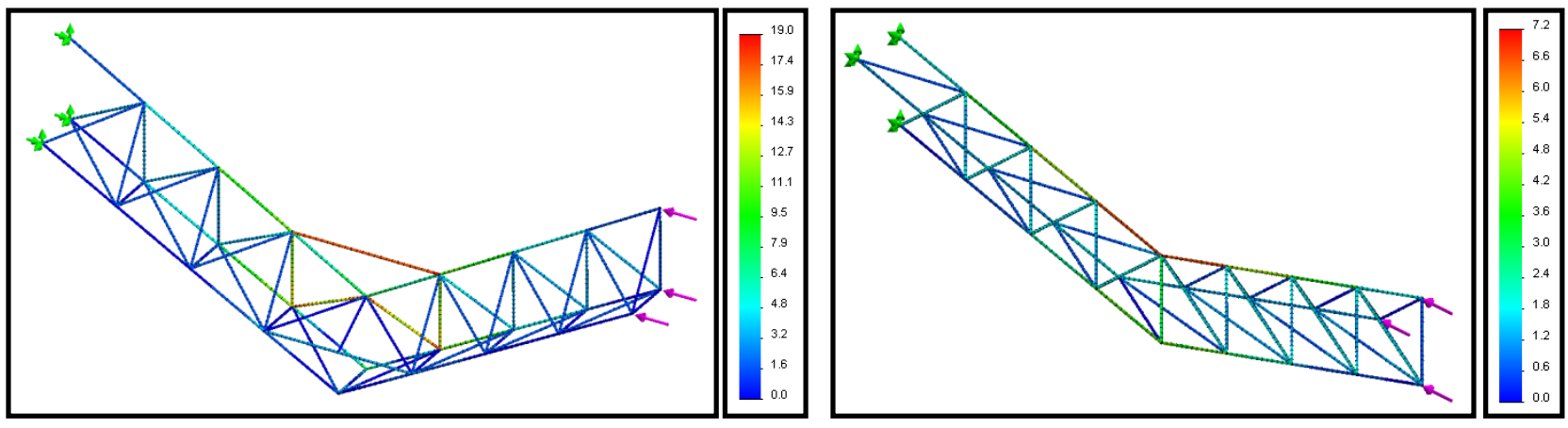

Figure 12. The images show stresses in the front (left) and rear (right) trusses when subjected to a total compressive force of $28 \mathrm{kN}$. The stress scales are listed in units of ksi. 
Initial analyses indicate that while the rear truss should be adequate to support this load with a safety factor of 5 , the front truss will not. Changes to the geometry of the middle bend in the truss, where it wraps around the HET structural Ibeam, will be required to minimize stress in this region. While some optimization will be required, overall the analysis shows that the sizing of the structural elements of the trusses should be adequate to support the structures, and that the weight estimate is on the correct order of magnitude.

\subsection{Enclosure Thermal Analysis}

Initial investigation into thermal management for the enclosures has resulted in baseline estimates for panel insulation thickness and coolant flow. Now that approximate spacing for the collimators has been determined, the next step will be a detailed CFD analysis using SolidWorks Flow Simulation. This will allow us to visualize the airflow and heat transfer through the enclosure, and provide data for optimization of fan sizing and ducting to eliminate hot spots. Once airflow requirements are determined, properly sized fan and coil units can be specified.

\section{SUMMARY AND PATH FORWARD}

Prior work done on the VIRUS support structure has clearly defined the design requirements for the final product. The concept presented in this paper shows promise in its capacity to meet these requirements. The enclosure location and free-standing VSS provide a current estimate of average IFU length of $23 \mathrm{~m}$. This exceeds the goal length, and investigation is ongoing into methods of further reducing that length, including alternate routing options from SR3 to the collimators, and adjusting the final enclosure location to bring it closer to SR3.

The next step in moving forward on the VSS will be detailing the enclosure design and lateral linkages. Thermal management of the enclosures will be undertaken first, as the location and size of air handling units and ductwork, as well as overall insulation thickness, plays a major role in the overall size of the enclosures and their footprint on the VSS. The lateral linkages, while not detailed in this concept, are integral to the design goal of sharing the azimuth drive of the HET.

The conceptual structure and enclosure design provides a total system mass of $25000 \mathrm{~kg}$, well under the maximum, and greatly reduces incursion into maintenance access regions of the telescope from previous designs. Preserving boom lift access through the front of the HET structure is paramount to sustaining the efficiency of the day-to-day operations at the HET, and avoiding costly downtime. This investigation of the concept has shown that it will be technically feasible to support VIRUS in a free-standing structure, and has opened the path for more detailed design work to follow.

\section{ACKNOWLEDGEMENT}

HETDEX is led by the University of Texas at Austin with participation from the Universitäts-Sternwarte of the LudwigMaximilians-Universität München, the Max-Planck-Institut für Extraterrestriche-Physik (MPE), Astrophysikalisches Institut Potsdam (AIP), Texas A\&M University, Pennsylvania State University, and the HET consortium. HETDEX is funded in part by gifts from Harold C. Simmons, Robert and Annie Graham, The Cynthia and George Mitchell Foundation, Louis and Julia Beecherl, Jim and Charlotte Finley, Bill and Bettye Nowlin, Robert and Fallon Vaughn, Eric Stumberg, and many others, by AFRL under agreement number FA9451-04-2-0355, and by the Texas Norman Hackerman Advanced Research Program under grants 003658-0005-2006 and 003658-0295-2007.

\section{REFERENCES}

[1] Hill, G.J., Gebhardt, K., Komatsu, E., Drory, N., MacQueen, P.J., Adams, J.A., Blanc, G.A., Koehler, R., Rafal, Roth, M.M., Kelz, A., Grupp, F., Murphy, J., Palunas, P., Gronwall, C., Ciardullo, R., Bender, R., Hopp, U., and Schneider, D.P., 2008, "The Hobby-Eberly Telescope Dark Energy Experiment (HETDEX): Description and Early Pilot Survey Results”, in Panoramic Views of the Universe, ASP Conf. Series, vol 399, p 115 (arXiv:0806.0183v1)

[2] R.D. Savage, et al., "Current status of the Hobby-Eberly Telescope wide field upgrade and VIRUS," Proc. SPIE, 7012-10 (2008)

[3] G.J. Hill, et al., "VIRUS: a massively replicated 33k fiber integral field spectrograph for the upgraded HobbyEberly Telescope,” Proc. SPIE, 7735-21 (2010) 
[4] H. Lee, et al., "LRS2: a new low-resolution spectrograph for the Hobby-Eberly Telescope and its application to scalable spectrographs for the future of extremely large telescopes,” Proc. SPIE, 7735-276 (2010)

[5] I. Soukup, et al., "Design of the fiber optic support system and fiber bundle accelerated life test for VIRUS," Proc. SPIE, 7735-180 (2010)

[6] H. Lee, et al., "VIRUS optical tolerance and production,” Proc. SPIE, 7735-140 (2010)

[7] J.L. Marshall, et al., "Production-line assembly of 150+ VIRUS spectrographs," Proc. SPIE, 7735-163 (2010)

[8] A. Kelz, et al., "Production and performance of replicable integral field units for VIRUS," Proc. SPIE, 7735-178 (2010)

[9] A. Collins, et al., "Development of VIRUS alignment assembly fixtures,” Proc. SPIE, 7735-263 (2010)

[10]B. Vattiat, et al., "Mechanical design evolution of the VIRUS instrument for volume production and deployment," Proc. SPIE, 7735-264 (2010)

[11]T.S. Chonis, et al., "Development of a cryogenic system for the VIRUS array of 150 spectrographs for the HobbyEberly Telescope,” Proc. SPIE, 7735-265 (2010)

[12] M.S. Worthington, et al., "Design and analysis of the Hobby-Eberly Telescope dark energy experiment (HETDEX) bridge,” Proc. SPIE, 7733-147 (2010)

[13] N.T. Mollison, et al., "Design and development of a long-travel positioning actuator and tandem constant force actuator safety system for the Hobby-Eberly Telescope wide field upgrade,” Proc. SPIE, 7733-150 (2010)

[14] G.A. Wedeking, et al., "Kinematic optimization of upgrade to the Hobby-Eberly Telescope through novel use of commercially available three-dimensional CAD package,” Proc. SPIE, 7733-148 (2010)

[15] J.J. Zierer, et al., "The development of high-precision hexapod actuators for the Hobby-Eberly Telescope dark energy experiment (HETDEX),” Proc. SPIE, 7733-49 (2010)

[16] J.R. Mock, et al., "Tracker controls development and control architecture for the Hobby-Eberly Telescope dark energy experiment,” Proc. SPIE, 7733-152 (2010)

[17]B.J. South, et al., "Wind loading analysis and strategy for deflection reduction on the HET Dark Energy Experiment upgrade,” Proc. SPIE, 7733-51 (2010)

[18] M.S. Worthington, et al., "Design and development of a high-precision, high-payload telescope dual-drive system," Proc. SPIE, 7733-201 (2010)

[19] N.T. Mollison, et al., "Collaborative engineering and design management for the Hobby-Eberly Telescope wide field upgrade,” Proc. SPIE, 7738-84 (2010)

[20]C. Ramiller, et al., "A new control system hardware architecture for the Hobby-Eberly Telescope prime focus instrument package,” Proc. SPIE, 7740-129 (2010)

[21]H. Lee, et al., "Analysis of active alignment control of the Hobby-Eberly Telescope wide field corrector using Shack-Hartmann wavefront sensors,” Proc. SPIE, 7738-18 (2010)

[22]H. Lee, et al., "Surface figure measurement of the Hobby-Eberly Telescope primary mirror segments via phase retrieval and its implications for the wavefront sensing in the new wide-field upgrade," Proc. SPIE, 7738-58 (2010)

[23] H. Lee, et al., "Orthonormal aberration polynominals over arbitrarily obscured pupil geometries for wavefront sensing in the Hobby-Eberly Telescope,” Proc. SPIE, 7738-59 (2010)

[24] H. Lee, et al., "Metrology systems for the active alignment control of the Hobby-Eberly Telescope wide-field upgrade,” Proc. SPIE, 7739-28 (2010)

[25] J. Good, et al., "Design of performance verification testing for HET wide-field upgrade tracker in the laboratory," Proc. SPIE, 7739-152 (2010)

[26] R. Savage, et al., "Current Status of the Hobby-Eberly Telescope wide field upgrade,” Proc. SPIE, 7733-149 (2010)

[27] A. Kelz, M. M. Roth, S. M. Bauer, B. Vattiat, E. Popow, Y. Padila, W. N. Rambolda, , M. Denke, R. Haynes, G. J. Hill, J. M. Good, J. D. Murphy, R. Savage, I. Soukup, N. Mollison, "Production and performance of replicable Integral-Field Units for VIRUS”, Proc. SPIE, 7735-178 (2010)

[28] J. H. Beno, J.A. Booth, J. R. Mock, "An alternative architecture and control strategy for hexapod positioning systems to simplify structural design and improve accuracy “, Proc. SPIE, 7733-97 (2010)

[29] J. Good, et al., “Current Status of the HETDEX Fiber Optic Support System,” Proc. SPIE 7014, 70147L (2008) 